1 Full title: THE TRYPANOSOME ALTERNATIVE OXIDASE: A POTENTIAL DRUG

TARGET?

3

4 Authors: Stefanie K Menzies, Lindsay B Tulloch, Gordon J Florence and Terry K

Smith

6

7 Postal Address: Biomedical Sciences Research Complex, University of St Andrews, St Andrews, Fife, Scotland KY16 9ST

9

Running Title: TAO: A Potential Drug Target?

11

12 Corresponding author: Terry K Smith, Biomedical Sciences Research Complex,

13 University of St Andrews, St Andrews, Fife, Scotland KY16 9ST 01334463412 
28 New drugs against Trypanosoma brucei, the causative agent of Human African

29 Trypanosomiasis, are urgently needed to replace the highly toxic and largely 30 ineffective therapies currently used. The trypanosome alternative oxidase (TAO) is an essential and unique mitochondrial protein in these parasites and is absent from

32 mammalian mitochondria, making it an attractive drug target. The structure and

33 function of the protein are now well characterized, with several inhibitors reported in

34 the literature which show potential as clinical drug candidates. In this review we 35 provide an update on the functional activity and structural aspects of TAO. We then 36 discuss TAO inhibitors reported to date, problems encountered with in vivo testing of 37 these compounds, and discuss the future of TAO as a therapeutic target.

Key Words: Trypanosome Alternative Oxidase, Drug Discovery, Chemotherapy, 
INTRODUCTION

Up to 70 million people in sub-Saharan Africa are at risk of contracting Human African Trypanosomiasis (HAT) (Simarro et al. 2012), also known as African Sleeping Sickness, caused by the kinetoplastid parasite Trypanosoma brucei. Two subspecies of the parasite cause disease in humans; T. brucei gambiense in West Africa and T. brucei rhodesiense in East Africa, both of which are spread by the tsetse fly. Both forms are fatal if untreated and are estimated to cause up to 20,000 cases of HAT per year (World Health Organization 2013). T. brucei evades the mammalian host immune system by changing their major surface coat proteins, known as variant surface glycoproteins (VSG), prior to each wave of host antibodies raised against the previous VSG type. Due to this sophisticated immune evasion technique known as antigenic variation, a vaccine against the disease is unlikely in the near future. Drugs currently in clinical use are associated with severe adverse effects, difficult administration, and increasing concerns regarding drug resistance.

64 Therefore, new drugs are urgently required (Lüscher et al. 2007). The drugs indicated for treatment of the disease (Figure 1) depend upon the subspecies of parasite and stage of the disease.

67

68 Early stage T. b. gambiense is treated with pentamidine, a diamidine hypothesized to act as a trypanocidal agent through several mechanisms, including disruption of the nucleus, kinetoplast and mitochondrial membrane potential (Baker et al. 2013). Late stage T. b. gambiense is treated with a combinational therapy of nifurtimox and eflornithine. Eflornithine is the only drug for HAT with a defined target, the ornithine 
73 decarboxylase, but the drug has poor potency against $T$. brucei and combination

74 therapy is required to prevent drug resistance acquired by loss of the drug uptake 75 transporter (Barrett \& Croft 2012). Suramin is recommended only for early stage $T$. 76 b. rhodesiense due to its inability to penetrate the blood brain barrier. Although the 77 mechanism of uptake by the parasites is known, the trypanocidal mode of action still 78 remains to be determined (Barrett \& Croft 2012, Zoltner et al. 2016). The arsenicalbased drug melarsoprol is recommended for late stage $T . b$. rhodesiense due to its ability to cross the blood brain barrier, however this property creates the often fatal adverse effect of encephalopathy in up to $10 \%$ of patients treated with the drug 82 (Kuepfer et al. 2012).

83 Differences in the biochemical processes between mammalian and trypanosomatid mitochondria make the mitochondrion an attractive drug target. One main difference between $T$. brucei and mammalian mitochondrial respiration is the presence of the trypanosome alternative oxidase (TAO), an essential non-cytochrome terminal

87 oxidase which has been extensively characterized as a drug target. This review will 88 summarize the structure and function of TAO and discuss the current progress 89 towards the development of inhibitors against this protein.

STRUCTURE AND FUNCTION OF THE TRYPANOSOME ALTERNATIVE

92 OXIDASE

93 Function

94 In 1960, Grant and Sargent first described the glycerol-3-phosphate oxidase (GPO) system as a cyanide-insensitive, oxygen-dependent mechanism of respiration in

96 Trypanosoma brucei rhodesiense (Grant \& Sargent 1960). The GPO system 97 consists of two enzymes; a mitochondrial FAD+-dependent glycerol-3-phosphate 
dehydrogenase (mG3PDH) and a terminal oxidase they termed the glycerol-3phosphate oxidase. Clarkson et al (Clarkson et al. 1989) proved that ubiquinol links the dehydrogenase and oxidase of the GPO system by acting as an electron carrier, and proposed that glycerol-3-phosphate oxidase was similar to the plant alternative

102 oxidase (AOX) and therefore should be renamed the trypanosome alternative 103 oxidase (TAO). The GPO system is responsible for the cyanide-insensitive oxygen104 dependent respiration in bloodstream form T. brucei, where the GPO shuttle facilitates the reoxidation of $\mathrm{NADH}$ to $\mathrm{NAD}^{+}$required for glycolysis. As shown in

106 Figure 2, the mG3PDH oxidizes glycerol-3-phosphate (Gly-3-P) to dihydroxyacetone 107 phosphate (DHAP), during which four electrons are transferred to ubiquinol. The 108 electrons from ubiquinol are subsequently oxidized by TAO to convert dioxygen into water. Alternative oxidases are found across a broad range of organisms, including 110 plants, nematodes, algae, yeast and T. brucei, but, curiously, are not known to be

111 present in the other human-infective trypanosomatids such as T. cruzi or Leishmania 112 spp.

113 TAO was first identified in Trypanosoma brucei by Chaudhuri et al (Chaudhuri et al. 114 1995) using antibodies against the alternative oxidase from Sauromatum guttatum, 115 which detected a $33 \mathrm{kDa}$ protein in the parasite's mitochondria. This $33 \mathrm{kDa}$ protein 116 was subsequently purified from bloodstream form T. brucei mitochondria and 117 confirmed to have ubiquinol oxidase activity. Chaudhuri et al (Chaudhuri et al. 118 1998) found that bloodstream form T. brucei express TAO 100-fold more than 119 procyclic form, which is believed to be due to the ability of procyclic forms to express 120 Complexes III and IV for ATP production via oxidative phosphorylation. Using areas

121 of high conservation in plant alternative oxidases, primers were designed to amplify 122 TAO from T. brucei gDNA (Chaudhuri \& Hill 1996). This enabled the identification of 
123 the single copy TAO gene (Tb927.10.7090) and subsequent cloning of TAO for

124 recombinant expression in Escherichia coli (Chaudhuri \& Hill 1996). Recombinant

125 TAO (rTAO) was subsequently used to determine the functional activity, kinetics and

126 inhibitors of the enzyme. Due to the endogenous ubiquinol oxidase activity of $E$. coli

127 by the cytochrome bo and bd complexes, it was necessary to perform these

128 investigations using hemA mutant $E$. coli, which are unable to synthesize the heme

129 necessary for cytochrome assembly. The ability of rTAO to restore respiration in

130 these cells showed the ability of TAO to function as a cyanide-insensitive terminal

131 oxidase. Research of rTAO by the Kita group established protocols for the

132 overproduction, solubilization and purification of rTAO for use in kinetic, structural

133 and inhibitor studies (Fukai et al. 1999; Fukai et al. 2003; Nihei et al. 2003; Yabu et

134 al. 2003).

135 TAO has been implicated in several other cellular activities, such as protection 136 against reactive oxygen species and regulation of surface protein expression. A role

137 of $A O X$ in photosynthetic plants is the rapid turnover of NADPH to protect the 138 photosynthetic machinery from radicals. It is possible that TAO has a related 139 function in $T$. brucei, to protect the rapidly metabolizing cells from damaging radicals.

140 The inhibition of TAO has been shown to induce oxidative damage to proteins and 141 increase production of reactive oxygen species (Fang \& Beattie 2003). Similarly, 142 inhibition of the electron transport chain and exposure to hydrogen peroxide causes 143 an upregulation in the expression of TAO (Fang \& Beattie 2003). This protection 144 against oxidative damage may explain the ability of TAO to inhibit drug-induced 145 programmed cell death-like phenomena in T. brucei (Tsuda et al. 2006). Vassella et 146 al (Vassella et al. 2004) reported the effects of TAO inhibition on the expression 147 levels of the procyclin GPEET, a cell surface protein found in procyclic form $T$. 
148 brucei. In the presence of the TAO inhibitor salicylhydroxamic acid (SHAM), GPEET

149 levels were heavily reduced, leading the authors to hypothesize that the level of

150 GPEET expression may be linked to the activity levels of TAO. Later studies

151 showed that the expression of TAO influences the expression of GPEET, where a

152 downregulation of both proteins may be important in the adaptation of the parasite to

153 survive within the tsetse fly midgut (Walker et al. 2005).

Structure

156 Several structures of the alternative oxidase were proposed (Andersson \& Nordlund

157 1999; Berthold et al. 2000) prior to the publication of the crystal structure. Initially, 158 hydropathy plots suggested the alternative oxidases contain two conserved 159 transmembrane regions, however later studies by Andersson and Nordlund

160 (Andersson \& Nordlund 1999) suggested alternative oxidases are not 161 transmembrane proteins, but rather interfacial inner membrane proteins. This was 162 confirmed with the solving of the crystal structure of TAO (Shiba et al. 2013) which is 163 devoid of any transmembrane domains, and instead has a hydrophobic face to partially bury the protein into the membrane (Figure 3). The recent publication of the crystal structure (Shiba et al. 2013) should help in the design of improved TAO

166 inhibitors. Sequence analysis of T. b. brucei, T. b. gambiense and T. b. rhodesiense 167 showed that the amino acid sequence of TAO is identical in all three species, and 168 therefore studies on TAO inhibitors and its co-structures can be directly applied from 169 the common laboratory model T. b. brucei to the human disease-causing subspecies 170 (Nakamura et al. 2010).

171 Early studies on plant alternative oxidases revealed that they were inhibited by metal 172 chelators (Schonbaum et al. 1971), and subsequent investigations using electron 
173 paramagnetic resonance (EPR) (Berthold et al. 2002; Moore et al. 2008) and

174 inductively coupled plasma-mass spectrometry (ICP-MS) (Kido et al. 2010) showed

175 that the alternative oxidases contained a non-heme diiron catalytic core that was 176 essential for catalytic activity and released during enzyme inactivation. In the crystal

177 structure in its oxidized state, the two Fe (III) ions are coordinated in a distorted 178 square pyramidal geometry to four glutamate residues and a hydroxo-bridge (Figure

179 4). Two conserved histidines located nearby are also likely to be involved in Fe180 coordination in the reduced state, as determined through Fourier transform infrared 181 spectroscopy (FTIR) investigations (Marechal et al. 2009). Together, the two 182 histidines and two glutamates form part of the two ExxH iron-binding motifs which 183 are common to all AOX proteins and are required for activity (Chaudhuri et al. 1998; 184 Ajayi et al. 2002).

\section{Mechanism of Catalysis}

187 The structure of TAO in complex with ubiquinol has not yet been solved so 188 hypotheses regarding ubiquinol-binding have been made based upon the structure of TAO complexed with ascofuranone analog AF2779OH. Superposition of ubiquinol over $\mathrm{AF} 2779 \mathrm{OH}$ indicates that during catalysis a ubiquinol molecule is highly likely to occupy the same position. The molecules gain entry to the diiron active site through

192 a relatively short $(\sim 10 \AA)$ hydrophobic channel from the membrane-bound side of

193 TAO (Figure 4). In this position the aromatic head of ubiquinol is less then $4.4 \AA$ 194 from the diiron core and is capable of forming hydrogen bonds with Arg118, Cys119 and Tyr220, all of which may be involved directly in catalysis rather than purely 196 substrate binding. 
197 A mechanism of catalytic activity has been proposed (Moore et al. 2013 and Figure

198 5), which begins with the diiron core in a reduced state (i.e. as $\mathrm{Fe}(\mathrm{II} / \mathrm{II})$ bridged by a 199 hydroxide). Upon binding of molecular oxygen to the $\mathrm{Fe}(\mathrm{II} / \mathrm{II})$ diiron core (Figure 5A), 200 one iron passes an electron to an oxygen atom, forming a superoxo intermediate 201 comprising an oxygen radical joined to an Fe(II/III) core. The oxygen radical 202 immediately abstracts a hydrogen atom (proton plus electron) from ubiquinol, 203 yielding a ubisemiquinone and a hydroperoxo intermediate (Figure 5B). The unstable 204 intermediate then undergoes a rearrangement whereby the hydroperoxo loses its 205 proton and electron to the hydroperoxide bridge, which is then released as water 206 (Figure 5C). The $\mathrm{Fe}(\mathrm{II} / \mathrm{II})$ core then gains an interaction with one of the histidines as 207 determined in FTIR experiments (Marechal et al. 2009) and the second atom of the 208 dioxygen forming a peroxodiiron. Homolytic cleavage of the O-O bond (Figure 5D) 209 yields an oxodiiron core, and one of the oxygens abstracts a hydrogen atom (proton 210 plus electron) from Tyr220 generating a tyrosyl radical, as observed by Marechal et 211 al (Marechal et al. 2009). The tyrosyl can then pick up an electron and proton from

212 the ubisemiquinone, either directly or via Cys119, releasing ubiquinone and returning 213 Tyr220 to its resting state. Moore's model suggests that ubiquinol in a second 214 channel can then provide two electrons and protons to release a second water and 215 reduce the diiron core back to its original $\mathrm{Fe}(\mathrm{II} / \mathrm{II})$ state bridged by a hydroxide ion 216 through an unknown mechanism (Figure 5E). However, the second ubiquinol 217 channel may not be needed as the release of ubiquinone creates the space for the 218 binding of a second ubiquinol in the same channel in a ping-pong binding fashion.

219 Furthermore, the mechanism of electron and proton transfer could proceed through a 220 similar route as for the first ubiquinol. 
223 The effectiveness of TAO inhibition to kill T. brucei has been well debated, with conflicting historical reports in the literature as to whether the inhibition of the GPO system alone is sufficient to kill the cells. As shown in Figure 2, bloodstream form $T$. brucei rely solely on glycolysis for ATP production, as opposed to the ATP-producing oxidative phosphorylation used by procyclic forms. In the presence of TAO inhibitors the oxidation of Gly-3-P to DHAP is blocked, causing an accumulation of Gly-3-P in the glycosome, which is converted to glycerol by the ATP-producing glycerol kinase (Yabu et al. 2006). This allows the recycling of glycosomal NAD+/NADH necessary 231 to continue glycolysis anaerobically.

232 Early reports of in vivo testing of TAO inhibitors suggested that although the compounds were able to inhibit the protein in vitro, this action alone was not sufficient to clear an infection when tested in animal models, due to anaerobic ATP production by the trypanosomes (Clarkson \& Brohn 1976; Grady et al. 1993; Yabu et al. 1998). It was believed that in order to cause cell death the anaerobic production of ATP also needed to inhibited with the co-administration of glycerol. However, later investigations showed that bloodstream T. brucei exposed to TAO inhibitors alone are unable to survive for more than 24 hours using only anaerobic respiration (Helfert et al. 2001). Furthermore, subsequent studies of a TAO inhibitor with an optimized dosing regimen but in the absence of glycerol, showed that TAO inhibition alone is sufficient to clear an infection in vivo (Yabu et al. 2003), indicating that inhibition of TAO is indeed a valid drug target.

244 There are few compounds that have been shown to be inhibitors of TAO. These compounds (Figure 6) all show structural similarity to the TAO substrate ubiquinol and are thought to act as competitive inhibitors, by binding to the ubiquinol binding 
247 site.

Salicylhydroxamic Acid

250 The first compounds to be investigated as TAO inhibitors were the aromatic 251 hydroxamates, such a salicylhydroxamic acid (SHAM) (Figure 6). SHAM was known 252 to be a potent inhibitor of the alternative oxidase in plants prior to the discovery of 253 the GPO system in trypanosomes, hence the compound was investigated as a 254 potential inhibitor of TAO. It is thought that hydroxamic acids compete with ubiquinol 255 for binding to $\mathrm{TAO}$, and thus the compounds prevent the translocation of electrons 256 from ubiquinol to oxygen (Pollakis et al. 1995). SHAM was found to have moderate $257\left(\mathrm{EC}_{50}=15 \mu \mathrm{M}\right)$ activity against $T$. brucei in vitro and was shown to specifically inhibit all TAO activity at $1 \mathrm{mM}$ (Opperdoes et al. 1976), although only a little effect was seen on ATP production. However, when the trypanocidal effect of SHAM was

260 investigated in vivo, the compound was unable to clear an infection and was only shown to be trypanocidal when co-administered with glycerol (Clarkson \& Brohn 1976).

SHAM is a poor clinical candidate, due to its low solubility in water (Nihei et al. 2002), which impairs the compounds from crossing the blood brain barrier, a critical characteristic required for drugs to effectively treat HAT. Numerous attempts were made to improve the potency of hydroxamic acids against TAO, but were unable to match the potency of SHAM when tested in vivo (Grady et al. 1993). Recently this issue has been revisited, Ott et al (Ott et al. 2006) developed novel SHAM analogs to improve its potency and solubility. SHAM analogs such as ACD16 (Figure 6) were designed to include a prenyl side chain, as found in the TAO substrate ubiquinol, and a carbohydrate group to improve solubility, whilst keeping the 2-hydroxybenzoic acid 
272 found in SHAM which is essential for TAO inhibition. These modifications lead to the

273 development of three compounds with up to five-fold greater potency than SHAM

274 against rTAO, however in vitro testing against $T$. b. brucei growth and respiration

275 revealed none of the modified compounds were more potent than SHAM. There

276 have been no subsequent reports on SHAM as a TAO inhibitor, although recent

277 reports on the efficacy of TAO inhibitors without glycerol (Yabu et al. 2003) may

278 renew interest in attempts to improve upon this compound.

\section{3,4-Dihydroxybenzoic Acid}

281 3,4-dihydroxybenzoic acids (Figure 6) were synthesized and tested as alternative 282 inhibitors of TAO, and displayed higher inhibitory activity than SHAM when tested in vitro, but this high potency was lost when the compounds were tested in vivo (Grady et al. 1993). To improve the bioavailability of the compounds, a series of $\mathrm{N}$ - $n$-alkyl3,4-dihydroxybenzamides were synthesized to increase solubility and decrease hydrolysis by serum esterases (Grady et al. 1993). Structure activity relationships of this series of compounds showed increasing potency and decreasing solubility as the length of the alkyl substituent increases. From this, $N$-n-butyl-3,4dihydroxybenzamide progressed to in vivo studies, and was found to effectively cure mice, but only when administered in conjunction with high doses of glycerol (450

$291 \mathrm{mg} / \mathrm{kg}$ drug with $15 \mathrm{~g} / \mathrm{kg}$ glycerol). Similar to SHAM, the high amount of glycerol

292 necessary for a trypanocidal effect of $\mathrm{N}$-n-butyl-3,4-dihydroxybenzamide rendered 293 the compound unfavourable as a clinical drug candidate, and no work has been undertaken to identify if an optimized dosing regimen might clear infection in vivo without glycerol. 
298 Ascofuranone (Figure 6), is a biologically active natural product isolated from the 299 fungus Ascochyta viciae. Minagawa et al first showed that ascofuranone is a potent 300 inhibitor of mitochondrial respiration of $T$. b. brucei, specifically the glucose- and 301 glycerol-3-phosphate-dependent respiration (Minagawa et al. 1997). Despite its high 302 potency against TAO, ascofuranone was initially found to only be trypanocidal in the presence of glycerol, similar to the other TAO inhibitors. The minimum inhibitory concentration of ascofuranone alone was $250 \mu \mathrm{M}$, whereas in the presence of $4 \mathrm{mM}$ glycerol potency was improved several thousand-fold to $30 \mathrm{nM}$ (Minagawa et al. 1997). Initially in vivo testing using mouse models found that ascofuranone was only curative when co-administered with a large amount $(3 \mathrm{~g} / \mathrm{kg})$ of glycerol (Yabu et al. 1998). Despite these less than favourable initial results, the dosage of ascofuranone was improved to once again render it a promising clinical drug candidate. Yabu et al

310 (Yabu et al. 2003) trialled the optimal dosage to cure T. b. brucei mice without glycerol and determined that $100 \mathrm{mg} / \mathrm{kg}$ intraperitoneally for 4 days and $400 \mathrm{mg} / \mathrm{kg}$

312 orally for 8 days completely cleared an infection, with a 50\% lethal dose (LD50) of

$313>1.2 \mathrm{~g} / \mathrm{kg}$ over 8 days. This study also provided evidence of the effects of 314 ascofuranone treatment on TAO, finding that ascofuranone decreased TAO activity 315 by $30 \%$ and increased the level of TAO expression within the cells (Yabu et al. 316 2003).

317 Ascofuranone was also shown to inhibit the TAO of T. vivax, which causes animal 318 trypanosomiasis (Nagana) in cattle. The T. vivax TAO has $76 \%$ identical amino acid 319 residues to $T$. brucei TAO (Suzuki et al. 2004) and the recombinant protein was 320 shown to be three-fold more sensitive to ascofuranone. Subsequent in vivo testing 321 of ascofuranone in T. vivax infected mice found that a single intramuscular dose of 
$32250 \mathrm{mg} / \mathrm{kg}$ ascofuranone without glycerol was sufficient to clear an infection, which

323 could be reduced still further to $6 \mathrm{mg} / \mathrm{kg}$ over 4 days whilst retaining $100 \%$ cure rate

324 within 48 hours. The high efficacy of ascofuranone against $T$. vivax may make this

325 compound a suitable drug for use against animal trypanosomiasis.

326 Kinetic analysis of ascofuranone inhibition of rTAO indicated a competitive

327 mechanism of inhibition against ubiquinol (Nihei et al. 2003). Recent studies of

328 ascofuranone have revealed the mechanism of inhibition, interaction with TAO and

329 the pharmacophore responsible for the inhibitory activity of ascofuranone (Saimoto

330 et al. 2013). The length of the linker chain between the aromatic ring and furanone

331 ring was shown to be important for its inhibitory activity, where the potency of

332 inhibitor with a propyl linker was a thousand-fold lower compared to nonyl and decyl

333 linkers. This is likely due to the interactions between the prenyl tail and membrane

334 lipid bilayers, where hydrophobicity of the inhibitor is influenced by the length of the

335 prenyl tail, which is important to access the membrane-associated TAO (Mogi et al.

336 2009; Saimoto et al. 2013). Attempts to improve the potency and selectivity of

337 ascofuranone-like analogs have been reported, such as the prenylphenol LL-Z1272

338 series by Mogi et al (Mogi et al. 2009) (Figure 6), although no results from in vivo

339 testing have been reported to date.

340

341 Aurachin D

342 Recently the natural product Aurachin D (Figure 6), a ubiquinol oxidase inhibitor

343 isolated from the bacterium Stigmatella aurantiaca strain Sg a15, was shown to have

344 inhibitory activity against T. b. gambiense (Li et al. 2013). Aurachin D is a mimic of

345 ubiquinol, with a quinolone core and prenyl chain. Li et al (Li et al. 2013) found that

346 Aurachin $\mathrm{D}$ inhibited $T$. b. gambiense with an $\mathrm{IC}_{50}$ of $1 \mu \mathrm{M}$, with a selectivity index 
347 greater than 35. Various analogs of Aurachin D were synthesized and tested for

348 trypanocidal activity, but none were improved compared to the natural product and

349 hence the compound has not been taken forward into animal models.

\section{CONCLUSIONS AND FUTURE PERSPECTIVES}

352 Although drugs against TAO have been studied for over 40 years, there are still no 353 drug candidates approaching clinical trials. The search for an effective TAO inhibitor

354 has been hampered until recently by the difficulty in obtaining a crystal structure of 355 the relatively unstable purified protein, and the historical conflicting reports on 356 whether inhibition of TAO alone is sufficient to kill T. brucei in vivo. However, recent 357 evidence renews the idea of TAO as a valid drug target. Although there are few 358 inhibitors of TAO reported in the literature, it is hoped that the publication of the 359 crystal structure of TAO will significantly improve the design of novel, potent 360 inhibitors against the enzyme. Further work is also still required to confirm the 361 mechanism of electron transfer by TAO and that ubiquinol is the true native co362 factor. 


\section{Acknowledgements}

364 We would like to thank the rest of the members of the Gordon Florence and Terry 365 Smith research groups.

366

367 Financial Support

368

369 This work was supported by the Leverhulme Trust (Grant number RL-2012-025) 
371 Ajayi, W., Chaudhuri, M. and Hill, G.C. 2002. "Site-Directed Mutagenesis Reveals 372 the Essentiality of the Conserved Residues in the Putative Diiron Active Site of the 373 Trypanosome Alternative Oxidase." Journal of Biological Chemistry 277 (10): 8187374 93. doi:10.1074/jbc.M111477200.

375

Andersson, M.E. and Nordlund, P. 1999. "A Revised Model of the Active Site of Alternative Oxidase." FEBS Letters 449: 17-22. doi:S0014-5793(99)00376-2 [pii].

Baker, N., de Koning, H.P., Mäser, P. and Horn, D. 2013. "Drug Resistance in 380 African Trypanosomiasis: The Melarsoprol and Pentamidine Story." Trends in 381 Parasitology 29 (3): 110-18. doi:10.1016/j.pt.2012.12.005.

382

383 Barrett, M.P., and Croft, S.L. 2012. "Management of Trypanosomiasis and 384 Leishmaniasis." British Medical Bulletin 104 (1): 175-96. doi:10.1093/bmb/lds031.

386 Berthold, D.A., Andersson, M.E. and Nordlund, P. 2000. "New Insight into the

387 Structure and Function of the Alternative Oxidase." Biochimica et Biophysica Acta 388 1460 (2-3): 241-54. doi:10.1016/S0005-2728(00)00149-3.

390 Berthold, D.A., Voevodskaya, N., Stenmark, P., Gräslund, A. and Nordlund, P. 391 2002. "EPR Studies of the Mitochondrial Alternative Oxidase: Evidence for a Diiron

392 Carboxylate Center." Journal of Biological Chemistry 277 (46): 43608-14. 393 doi:10.1074/jbc.M206724200. 
395 Bringaud, F., Barrett, M.P. and Zilberstein, D. 2012. "Multiple Roles of Proline

396 Transport and Metabolism in Trypanosomatids." Frontiers in Bioscience 17: 349-74.

397

398 Chaudhuri, M., Wilfred A. and Hill, G.C.. 1998. "Biochemical and Molecular

399 Properties of the Trypanosoma Brucei Alternative Oxidase." Molecular and 400 Biochemical Parasitology 95: 53-68.

401

402 Chaudhuri, M., Wilfred, A., Temple, S. and Hill, G.C. 1995. "Identification and 403 Partial Purification of a Stage-Specific $33 \mathrm{kDa}$ Mitochondrial Protein as the 404 Alternative Oxidase of the Trypanosoma Brucei Brucei Bloodstream 405 Trypomastigotes." The Journal of Eukaryotic Microbiology 42 (5): 467-72.

406

407 Chaudhuri, M., and Hill, G.C. 1996. "Cloning, Sequencing and Functional Activity of 408 the Trypanosoma Brucei Brucei Alternative Oxidase." Molecular and Biochemical 409 Parasitology 83: 125-29.

410

411 Clarkson, A.B. and Brohn, F.H. 1976. "Trypanosomiasis: An Approach to

412 Chemotherapy by the Inhibition of Carbohydrate Catabolism." Science (New York, 413 N.Y.) 194 (4261): 204-6. doi:10.1126/science.986688.

415 Clarkson, A.B., Bienensb, E.J., Pollakisz, G. and Gradyll, W. 1989. "Respiration 416 of Bloodstream Forms of the Parasite Trypanosoma Brucei Brucei Is Dependent on 417 a Plant-like Alternative Oxidase." The Journal of Biological Chemistry 264 (30): $418 \quad 17770-76$. 
420 Fang, J., and Beattie, D.S. 2003. "Alternative Oxidase Present in Procyclic

421 Trypanosoma Brucei May Act to Lower the Mitochondrial Production of Superoxide." 422 Archives of Biochemistry and Biophysics 414: 294-302. doi:10.1016/S0003$4239861(03) 00196-6$.

425 Fukai, Y., Amino, H., Hirawake, H., Yabu, Y., Ohta, N., Minagawa, N., Sakajo, S., 426 Yoshimoto, A., Nagai, K., Takamiya, S., Kojima, S. and Kita, K. 1999. "Functional 427 Expression of the Ascofuranone-Sensitive Trypanosoma Brucei Brucei Alternative 428 Oxidase in the Cytoplasmic Membrane of Escherichia Coli." Comparative 429 Biochemistry and Physiology. Part C, Pharmacology, Toxicology \& Endocrinology 430124 (2): 141-48. doi:10.1016/S0742-8413(99)00040-7.

431

432 Fukai, Y., Coichi N., Keisuke K., Yoshisada Y. and Suzuki, T. 2003. 433 "Overproduction of Highly Active Trypanosome Alternative Oxidase in Escherichia 434 Coli Heme-Deficient Mutant." Parasitology International 52: 237-41. 435 doi:10.1016/S1383-5769.

436

437 Grady, R.W., Bienen, E.J., Dieck, H.A., Saric, M. and Clarkson, A.B. 1993. “N-N438 Alkyl-3,4-Dihydroxybenzamides as Inhibitors of the Trypanosome Alternative 439 Oxidase: Activity in Vitro and in Vivo." Antimicrobial Agents and Chemotherapy 37 $440 \quad$ (5): 1082-85. doi:10.1128/AAC.37.5.1082.

441

442 Grant, P.T. and Sargent, J.R. 1960. "Properties of L-Alpha-Glycerophosphate 443 Oxidase and Its Role in the Respiration of Trypanosoma Rhodesiense." The 444 Biochemical Journal 76: 229-37. 
446 Helfert, S., Estévez, A.M., Bakker, B., Michels, P. and Clayton, C. 2001. "Roles of

447 Triosephosphate Isomerase and Aerobic Metabolism in Trypanosoma Brucei." The 448 Biochemical Journal357 (Pt 1): 117-25. doi:10.1042/0264-6021:3570117.

450 Kido, Y., Shiba, T., Inaoka, D.K., Sakamoto, K., Nara, T., Aoki, T., Honma, T., 451 Tanaka, A., Inoue, M., Matsuoka, S., Moore, A., Harada, S. and Kita, K. 2010. "Crystallization and Preliminary Crystallographic Analysis of Cyanide-Insensitive Alternative Oxidase from Trypanosoma Brucei Brucei." Acta Crystallographica. 454 Section F, Structural Biology and Crystallization Communications 66 (Pt 3). 455 International Union of Crystallography: 275-78. doi:10.1107/S1744309109054062.

Kuepfer, I., Schmid, C., Allan, M., Edielu, A., Haary, E.P., Kakembo, A., Kibona,

S., Blum, J. and Burri, C. 2012. "Safety and Efficacy of the 10-Day Melarsoprol Schedule for the Treatment of Second Stage Rhodesiense Sleeping Sickness." $460 \quad$ PLoS Neglected Tropical Diseases 6 (8). doi:10.1371/journal.pntd.0001695.

Li, X.W., Herrmann, J., Zang, Y., Grellier, P., Prado, S., Muller, R. and Nay, B. 2013. "Synthesis and Biological Activities of the Respirator Chain Inhibitor Aurachin D and New Ring versus Chain Analogues." Beilstein Journal of Organic Chemistry 9 (Figure 1): 1551-58. doi:10.3762/bjoc.9.176.

466

467 Lüscher, A., de Koning, H.P. and Mäser, P. 2007. "Chemotherapeutic Strategies 468 against Trypanosoma Brucei: Drug Targets vs. Drug Targeting." Current 469 Pharmaceutical Design 13 (April): 555-67. doi:10.2174/138161207780162809. 
471 Marechal, A., Kido, Y., Kita, K., Moore, A.L. and Rich, P.R. 2009. "Three Redox

472 States of Trypanosoma Brucei Alternative Oxidase Identified by Infrared 473 Spectroscopy and Electrochemistry." The Journal of Biological Chemistry 284 (46): 474 31827-33. doi:10.1074/jbc.M109.059980.

475

476 Minagawa, N., Yabu, Y., Kita, K., Nagai, K., Ohta, N., Meguro, K., Sakajo, S.and 477 Yoshimoto, A. 1997. "An Antibiotic, Ascofuranone, Specifically Inhibits Respiration 478 and in Vitro Growth of Long Slender Bloodstream Forms of Trypanosoma Brucei 479 Brucei." Molecular and Biochemical Parasitology 81 (2): 127-36. doi:10.1016/0166$480 \quad 6851(96) 02665-5$.

481

Mogi, T., Ui, H., Shiomi, K., Omura, S., Miyoshi, H. and Kita, K. 2009. "Antibiotics 483 LL-Z1272 Identified as Novel Inhibitors Discriminating Bacterial and Mitochondrial 484 Quinol Oxidases.” Biochimica et Biophysica Acta - Bioenergetics 1787 (2): 129-33. 485 doi:10.1016/j.bbabio.2008.11.016.

486

Moore, A.L., Carré, J.E., Affourtit, C., Albury, M.S., Crichton, P.G., Kita, K. and

Heathcote, P. 2008. "Compelling EPR Evidence That the Alternative Oxidase Is a 489 Diiron Carboxylate Protein.” Article. Biochimica et Biophysica Acta 1777 (4): 327$490 \quad 330$. doi:10.1016/j.bbabio.2008.01.004.

491

492 Moore, A.L., Shiba, T.,Young, L., Harada, S., Kita, K. and Ito,K. 2013. “Unraveling 493 the Heater: New Insights into the Structure of the Alternative Oxidase." Annual 494 Review of Plant Biology 64 (January): 637-63. doi:10.1146/annurev-arplant-042811- 
497 Nakamura, K., Fujioka, S., Fukumoto, S., Inoue, N., Sakamoto, K., Hirata, H., 498 Kido, Y., Yabu, Y., Suzuki, T., Watanabe, Y.I., Saimoto, H., Akiyama, H. and Kita, 499 K. 2010. "Trypanosome Alternative Oxidase, a Potential Therapeutic Target for 500 Sleeping Sickness, Is Conserved among Trypanosoma Brucei Subspecies." 501 Parasitology International 59 (4). Elsevier Ireland Ltd: 560-64. 502 doi:10.1016/j.parint.2010.07.006.

504 Nihei, C., Fukai, Y., Kawai, K., Osanai, A., Yabu, Y., Suzuki, T., Ohta, N., 505 Minagawa, N., Nagai, K. and Kita, K. 2003. "Purification of Active Recombinant 506 Trypanosome Alternative Oxidase." FEBS Letters 538 (1-3): 35-40. 507 doi:10.1016/S0014-5793(03)00120-0.

509 Nihei, C., Fukai, Y. and Kita, K. 2002. "Trypanosome Alternative Oxidase as a 510 Target of Chemotherapy." Biochimica et Biophysica Acta - Molecular Basis of 511 Disease 1587 (2-3): 234-39. doi:10.1016/S0925-4439(02)00086-8.

513 Opperdoes, F.R., Borst, P. and Fonck, K. 1976. "The Potential Use of Inhibitors of

514 Glycerol-3-Phosphate Oxidase for Chemotherapy of African Trypanosomiasis." 515 FEBS Letters 62 (2): 169-72. doi:10.1016/0014-5793(76)80045-2.

517 Ott, R., Chibale, K., Anderson, S., Chipeleme, A., Chaudhuri, M., Guerrah, A.,

518 Colowick, N. and Hill, G.C. 2006. "Novel Inhibitors of the Trypanosome Alternative 519 Oxidase Inhibit Trypanosoma Brucei Brucei Growth and Respiration." Acta Tropica 
100 (3): 172-84. doi:10.1016/j.actatropica.2006.10.005.

522 Pollakis, G., Grady, R.W., Dieck, H.A. and Clarkson, A.B. 1995. "Competition

523 between Inhibitors of the Trypanosome Alternative Oxidase (TAO) and Reduced

524 Coenzyme Q9.” Biochemical Pharmacology 50 (8): 1207-10. doi:10.1016/0006$525 \quad 2952(95) 00259-3$.

526

527 Saimoto, H., Kido, Y., Haga, Y., Sakamoto, K. and Kita, K. 2013. "Pharmacophore 528 Identification of Ascofuranone, Potent Inhibitor of Cyanide-Insensitive Alternative 529 Oxidase of Trypanosoma Brucei." Journal of Biochemistry 153 (3): 267-73. 530 doi:10.1093/jb/mvs135.

531

532 Schonbaum, G.R., Bonner, W.D., Storey, B.T. and Bahr, J.T. 1971. "Specific 533 Inhibition of the Cyanide-Insensitive Respiratory Pathway in Plant Mitochondria by 534 Hydroxamic Acids." Plant Physiology 47 (1): 124-28. doi:10.1104/pp.47.1.124.

536 Shiba, T., Kido, Y., Sakamoto, K., Ken, D., Tsuge, C. and Tatsumi, R. 2013. 537 "Structure of the Trypanosome Cyanide-Insensitive Alternative Oxidase." 538 Proceedings of the National Academy of Sciences 110 (12): 4580-85. 539 doi:10.1073/pnas.1218386110/-

540 /DCSupplemental.www.pnas.org/cgi/doi/10.1073/pnas.1218386110.

542 Simarro, P.P., Cecchi, G., Franco, J.R., Paone, M., Diarra, A., Ruiz-Postigo, J.A., 543 Fèvre, E.M., Mattioli, R.C. and Jannin, J.G. 2012. "Estimating and Mapping the 544 Population at Risk of Sleeping Sickness." PLoS Neglected Tropical Diseases 6 (10): 
e1859. doi:10.1371/journal.pntd.0001859.

546

547 Suzuki, T., Nihei, C.I.,Yabu, Y., Hashimoto, T., Suzuki, M., Yoshida, A., Nagai,

548 K., Hosokawa, T., Minagawa, N., Suzuki, S., Kita, K. and Ohta, N. 2004.

549 "Molecular Cloning and Characterization of Trypanosoma Vivax Alternative Oxidase

550 (AOX) Gene, a Target of the Trypanocide Ascofuranone." Parasitology International 53 (3): 235-45. doi:10.1016/j.parint.2004.02.001.

552

Tsuda, A., Witola, W.H., Konnai, S., Ohashi, K. and Onuma, M. 2006. "The Effect of TAO Expression on PCD-like Phenomenon Development and Drug Resistance in

Trypanosoma

Brucei." Parasitology

International 55

(2): $\quad 135-42$. doi:10.1016/j.parint.2006.01.001.

Vassella, E., Probst, M., Schneider, A., Studer, E., Renggli, C.K., and Roditi, I. 2004. "Expression of a Major Surface Protein of Trypanosoma Brucei Insect Forms

560 Is Controlled by the Activity of Mitochondrial Enzymes." Molecular Biology of the Cell 561 15 (9): 3986-93. doi:10.1091/mbc.E04-04-0341.

562

563 Walker, R., Saha, L., Hill, G.C. and Chaudhuri, M. 2005. "The Effect of over-

564 Expression of the Alternative Oxidase in the Procyclic Forms of Trypanosoma

565 Brucei." Molecular and Biochemical Parasitology 139 (2): 153-62. doi:10.1016/j.molbiopara.2004.11.003.

568 World Health Organization. 2013. "Control and Surveillance of Human African 569 Trypanosomiasis." World Health Organization Technical Report Series, no. 984: 1- 
572 World Health Organization. 2016. "Human African Trypanosomiasis."

573 http://apps.who.int/gho/data/node.main.A1635?lang=en.

575 Yabu, Y., Minagawa, N., Kita, K., Nagai, K., Honma, M., Sakajo, S., Koide, T., 576 Ohta, N. and Yoshimoto, A. 1998. "Oral and Intraperitoneal Treatment of

577 Trypanosoma Brucei Brucei with a Combination of Ascofuranone and Glycerol in 578 Mice." Parasitology International 47 (2): 131-37. doi:10.1016/S1383-5769(98)000115797.

580

581 Yabu, Y., Suzuki, T., Nihei, C.I., Minagawa, N., Hosokawa, T., Nagai, K., Kita, K. and Ohta, N. 2006. "Chemotherapeutic Efficacy of Ascofuranone in Trypanosoma Vivax-Infected Mice without Glycerol." Parasitology International 55 (1): 39-43. doi:10.1016/j.parint.2005.09.003.

585

586 Yabu, Y., Yoshida, A., Suzuki, T., Nihei, C.I., Kawai, K., Minagawa, N.,

587 Hosokawa, T., Nagai, K., Kita, K. and Ohta, N. 2003. "The Efficacy of 588 Ascofuranone in a Consecutive Treatment on Trypanosoma Brucei Brucei in Mice." 589 Parasitology International 52 (2): 155-64.

Zoltner, M., Horn, D., de Koning, H.P., and Field, M.C. 2016. “Exploiting the Achilles' Heel of 

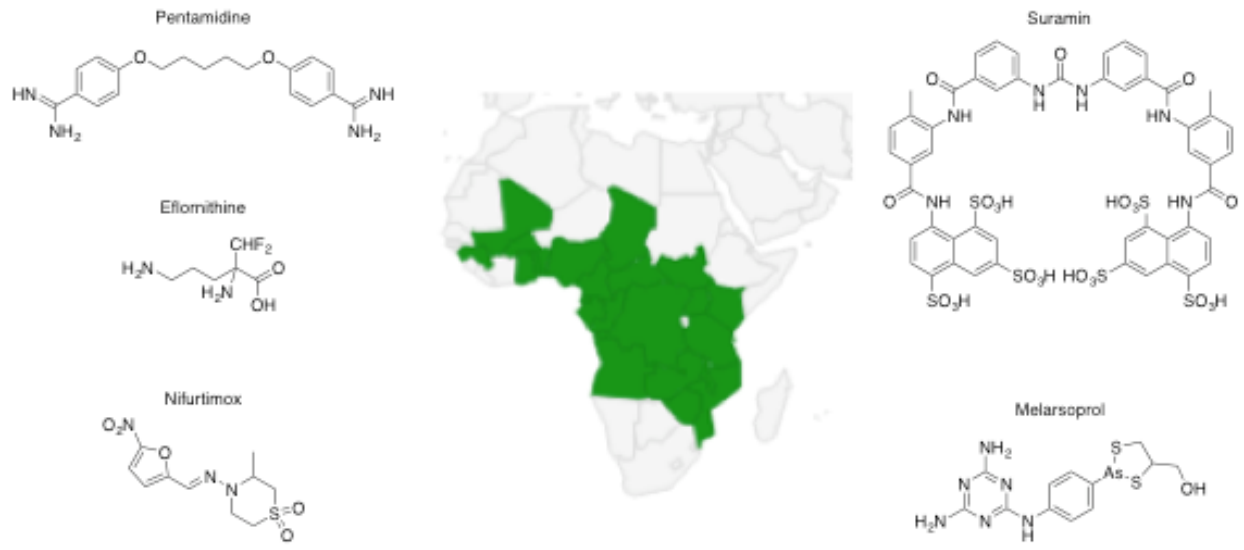

Figure 1. Distribution of countries endemic for Trypanosoma brucei according to WHO and the currently used clinical drugs 


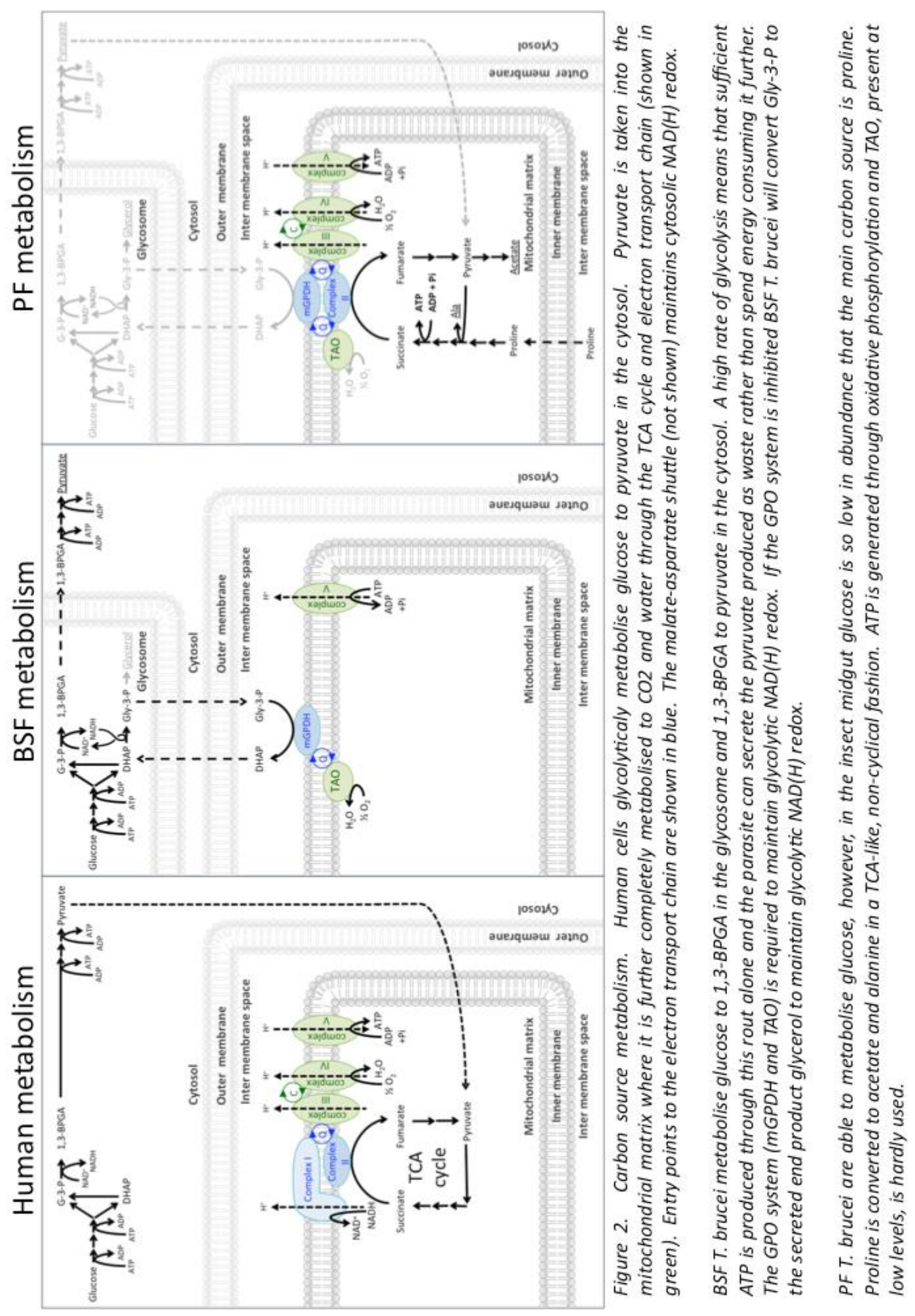




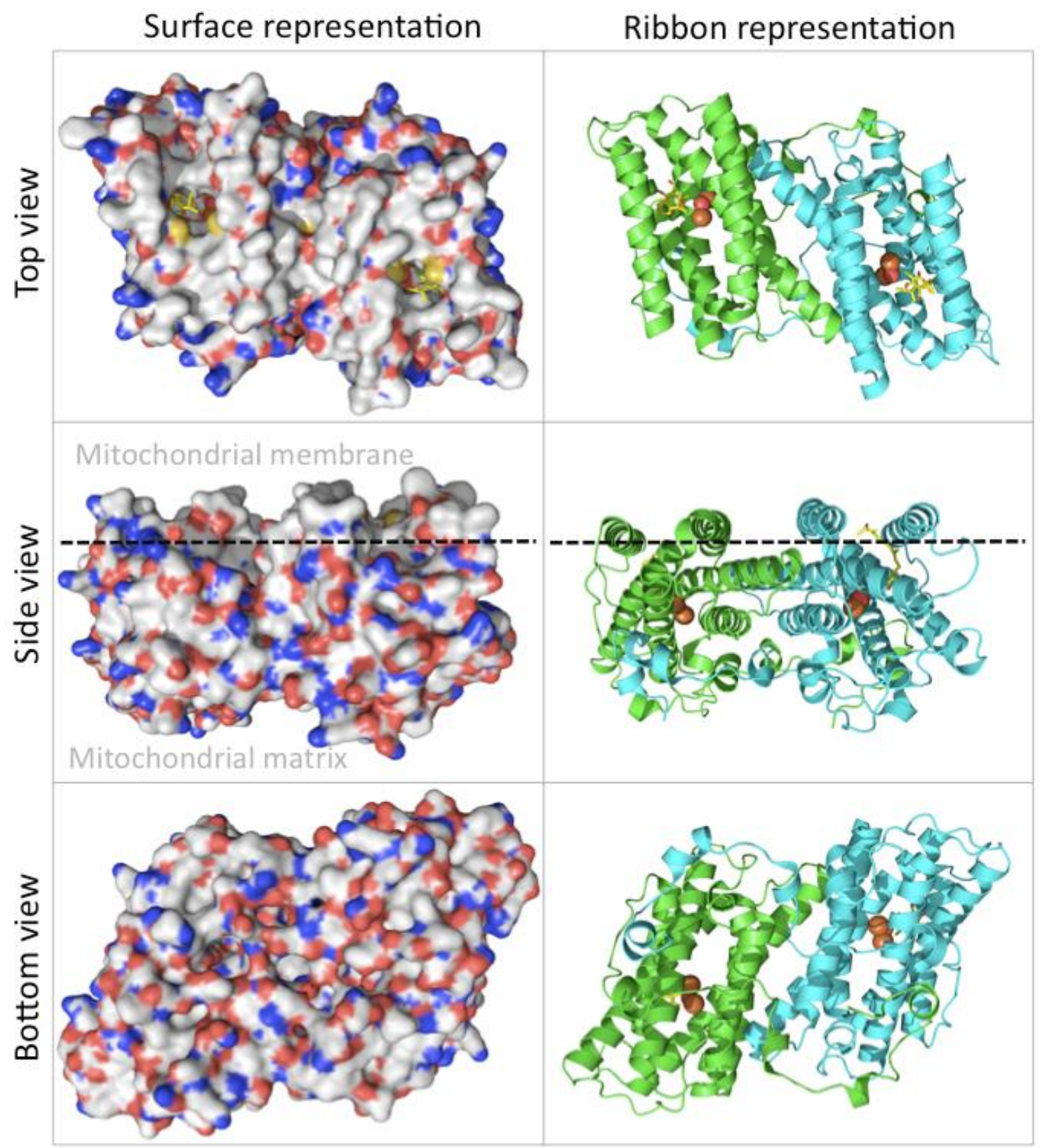

Figure 3. The TAO dimer in complex with diiron/hydroxo core (shown as spheres) and inhibitor $A F 27790 H$ (shown in yellow carbon stick). For surface representation, hydrophobic areas are grey and hydrophilic areas are blue/red. The upper face of the dimer is highly hydrophobic allowing TAO to burry itself within a single layer of the inner mitochondrial membrane. The approximate position of the membrane/matrix interface is represented by a dashed line. The diiron catalytic core is burried deep within the protein structure and a channel from the membrane to the core allows access of ubiquinol substrate (or analogous inhibitor AF2779OH). 


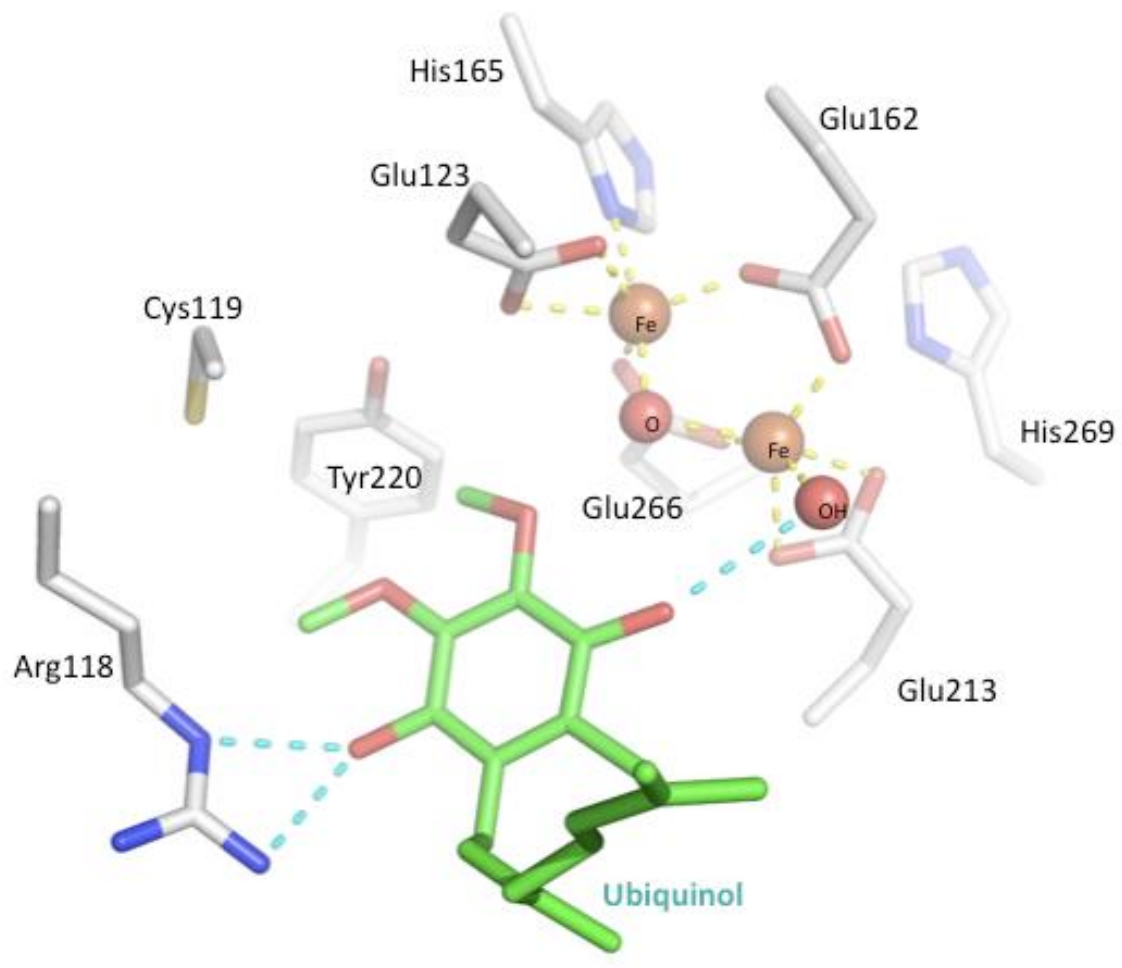

Figure 4. The TAO active site with ubiquinol (green) superposed in the place of inhibitor AF2779OH. The diiron core is in theFe(III/III) oxodiiron state (see mechanism part in the main body text), coordinated by four glutamates, two histidines, an oxygen and a hydroxyl (yellow dashed lines).

For the first ubiquinol oxidation, the coordinated $\mathrm{OH}$ would abstract a hydrogen atom (proton plus electron) from ubiquinol (blue dashed line) and leave as water, reducing the core to $\mathrm{Fe}(\mathrm{II} / \mathrm{III})$ and oxidising ubiquinol to semiubiquinol. The oxygen bridge will take a hydrogen from Tyr220 to form a hydroxo bridge and the resulting tyrosyl radical will take a hydrogen from semiubiquinol either directly or via Cys 119 and return to its native state, leaving the core in a reduced diferrous Fe(II/II) state bridged by a sinle hydroxyl. With the reaction complete, ubiquinone will leave allowing molecular oxygen and a second ubiquinol to enter. 
Ubiquinol<smiles>COC1=C(OC)C(=O)C(CC=C(C)C(C)C)=CC1=O</smiles>

Ascofuranone<smiles>CC(=CCc1c(O)c(Cl)c(C)c(C=O)c1O)CCC=C(C)C1CC(=O)C(C)(C)O1</smiles>

SHAM Search d<smiles>O=C(NO)c1ccccc1O</smiles>

3,4-dihydroxybenzoic Acid<smiles>O=C(O)c1ccc(O)c(O)c1</smiles>

Aurachin D<smiles>CC(C)=CCC/C(C)=C/CC/C(C)=C/Cc1c(C)[nH]c2ccccc2c1=O</smiles>

Figure 5. Chemical structures of TAO substrate ubiquinol and the TAO inhibitors salicylhydroxamic acid (SHAM), ascofuranone, 3,4-dihydroxybenzoic acid and Aurachin D. 
